\title{
Correction to: Malachite Green and Crystal Violet Decolorization by Ganoderma lucidum and Pleurotus ostreatus Supernatant and by rGILCC1 and rPOXA 1B Concentrates: Molecular Docking Analysis
}

\author{
Edwin D. Morales-Álvarez ${ }^{1,2}$ - Claudia M. Rivera-Hoyos ${ }^{3}$. \\ Sergio A. Poveda-Cuevas ${ }^{4}$ - Edwin A. Reyes-Guzmán ${ }^{5,6}$. \\ Aura M. Pedroza-Rodríguez ${ }^{1}$ - Edgar A. Reyes-Montaño ${ }^{5}$. \\ Raúl A. Poutou-Piñales ${ }^{3}$
}

Published online: 9 October 2017

(C) Springer Science+Business Media, LLC 2017

Correction to: Appl Biochem Biotechnol. https://doi.org/10.1007/s12010-017-2560-y

The original version of this article unfortunately contained a mistake. The replacement image of Fig. 4 provided by the first corresponding author, Aura M. Pedroza-Rodríguez, was

The online version of the original article can be found at http://dx.doi.org/10.1007/s12010-017-2560-y

Aura M. Pedroza-Rodríguez

apedroza@javeriana.edu.co

$\triangle$ Edgar A. Reyes-Montaño eareyesm@unal.edu.co

$\bowtie$ Raúl A. Poutou-Piñales rpoutou@javeriana.edu.co

1 Laboratorio de Microbiología Ambiental y Suelos, Grupo de Biotecnología Ambiental e Industria (GBAI), Departamento de Microbiología, Facultad de Ciencias, Pontificia Universidad Javeriana, Carrera 7ma No 43-82, Edifício 50 Lab. 106, Bogotá 110-23 DC, Colombia

2 Departamento de Química, Facultad de Ciencias Exactas y Naturales, Universidad de Caldas, Manizales-Caldas, Colombia

3 Laboratorio de Biotecnología Molecular, Grupo de Biotecnología Ambiental e Industrial (GBAI), Departamento deMicrobiología, Facultad de Ciencias, Pontificia Universidad Javeriana, Carrera $7 \mathrm{ma}$ No 43-82, Edifício 50 Lab. 124, Bogotá 110-23 DC, Colombia

4 Escuela de Ciencias Biológicas, Facultad de Ciencias Básicas, Universidad Pedagógica y Tecnológica de Colombia (UPTC), Tunja-Boyacá, Colombia 
incorrect and that the originally submitted Fig. 4 should have been retained provided below. The original article has been corrected.
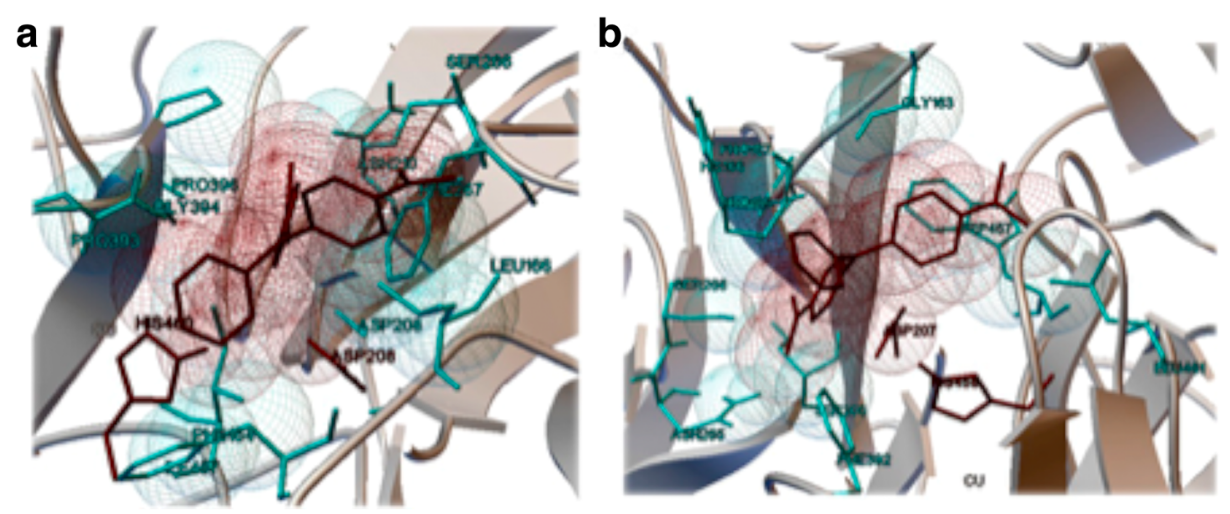

Fig. 4 MG interactions with the GILCC1 tape diagram (a) and POXA 1B (b) models. Interacting residues Van der Waals radius and MG radius are observed. Amino acid side chains are represented in stick models colored light blue. Dye and flexible residues are the stick models colored in red, where and the T1 copper position stands out. Interactions were visualized in AutoDock

5 Grupo de Investigación en Proteínas. Departamento de Química, Facultad de Ciencias, Universidad Nacional de Colombia (UNAL), Carrera 30 No 45-03 Edificio 451, oficina 458, Bogotá 110-01 DC, Colombia

6 Departamento de Química, Facultad de Ciencias, Universidad Antonio Nariño (UAN), Bogotá, DC, Colombia 